\title{
Preparation and Characterization of Cellulose Nanofibril/Polyvinyl Alcohol Composite Nanofibers by Electrospinning ${ }^{1}$
}

\author{
Byung-Dae Park $^{2 \dagger} \cdot$ In Chul $\mathrm{Um}^{3} \cdot$ Sun-Young Lee ${ }^{4} \cdot$ Alain Dufresne $^{5}$
}

\begin{abstract}
This work undertook to prepare nanofibers of cellulose nanofibrils (CNF)/polyvinyl alcohol (PVA) composite by electrospinning, and characterize the electrospun composite nanofibers. Different contents of CNFs isolated from hardwood bleached kraft pulp (HW-BKP) by 2,2,6,6-tetramethylpiperidine-1-oxy radical (TEMPO)-mediated oxidation were suspended in aqueous polyvinyl alcohol (PVA) solution, and then electrospun into CNF/PVA composite nanofibers. The morphology and dimension of CNFs were characterized by transmission electron microscopy (TEM), which revealed that CNFs were fibrillated form with the diameter of about $7.07 \pm 0.99 \mathrm{~nm}$. Morphology of the electrospun nanofiber observed by field-emission scanning electron microscopy (FE-SEM) showed that uniform CNF/PVA composite nanofibers were manufactured at $1 \sim 3 \% \mathrm{CNF}$ contents while many beads were observed at $5 \% \mathrm{CNF}$ level. Both the viscosity of CNF/PVA solution and diameter of the electrospun nanofiber decreased with an increase in CNF content. The diameter and its distribution of the electrospun nanofibers helped explain the differences observed in their morphology. These results show that the electrospinning method was successful in preparing uniform CNF/PVA nanofibers, indicating a great potential for manufacturing consistent and reliable cellulose-based nanofibrils for scaffolds in future applications.
\end{abstract}

Keywords : Cellulose nanofibril, TEMPO, Polyvinyl alcohol, Electrospinning, Nanofiber, Diameter distribution

\section{INTRODUCTION}

Among the natural biomaterials, cellulose is the most abundant, renewable and biodegradable polymer in the world. Cellulose fibrils consist of different hierarchical microstructures. Parallel and long cellulose chains are stabilized laterally by hydrogen bonds between hydroxyl groups, resulting in high structural strength and stiffness. The reported elastic modulus of native cellulose I crystal is $167.5 \mathrm{GPa}$ (Tashiro \& Kobayashi, 1991) and that of tunic in whiskers is $143 \mathrm{GPa}$ (Sturcováe et al., 2005). Because of its excellent properties, nanocellulose has gen-

1 Received December 27, 2013; accepted February 10, 2014

2 Department of Wood Science and Technology, Kyungpook National University, Daegu 702-701, Republic of Korea

3 Department of Bio-fibers and Materials Science, Kyungpook National University, Daegu 702-701, Republic of Korea

4 Division of Wood Processing, Korea Forest Research Institute, Seoul 130-712, Republic of Korea

5 Grenoble Institute of Technology (Grenoble INP), The International School of Paper, Print Media and Biomaterials (Pagora), CS10065, 38402 Saint Martin deHere, France

† Corresponding author : Byung-Dae Park (e-mail: byungdae@knu.ac.kr) 
erated a great deal of interest as nanometer size reinforcement agent for a range of biocomposites.

Advances in nanotechnology have attracted much attention with regard to the utilization of cellulose nanomaterials, such as cellulose nanowhiskers (CNWs), cellulose nanofibrils (CNFs), or crystalline cellulose particles for preparing high performance nanocomposites because highly crystalline cellulose-based nanofibers are environmentally compatible, and are endowed with several other unique and desirable features. Thus, cellulose-based nanomaterials are being increasingly employed for fabricating a wide variety of nanocomposites in combination with polymers, and the developments are benefitting from rapid advances in nanoscience and nanotechnologies (Azizi Samir et al., 2005; Eichhorn et al., 2010). Preparation and characterization related to CNFsbased nanocomposites have been extensively studied in anticipation of many desirable property contributions from CNFs, such as high strength, high stiffness, biodegradability and lightweight.

In the last two decades, the 2,2,6,6-tetramethylpiperidine-1-oxyradical (TEMPO), a watersoluble, commercially available and stable nitroxyl radical, has paved the way fora new field of conversion chemistry for efficient and selective conversion of alcoholic hydroxyl groups to aldehydes, ketones and carboxyl groups under mild conditions (de Nooy et al., 1995; de Nooy et al., 1996). Many related studies using TEMPO have been extensively carried out for CNFs from several wood pulps and cotton linters in the last few years (Fukuzumi et al., 2009; Saito et al., 2006; Saito et al., 2007; Isogai et al., 2011). CNFs prepared by TEMPO-mediated oxidation of native celluloses and successive mechanical disintegration of the oxidized celluloses in water have also been extensively used for fabricating nanocomposites (Isogai et al., 2011).

According to the TEMPO-mediated oxidation, the C6 primary hydroxyls of cellulose are oxidized to $\mathrm{C} 6$ carboxylate groups in water at $\mathrm{pH}$ $10 \sim 11$. The oxidation process can be monitored from the pattern of aqueous $\mathrm{NaOH}$ consumption, which is continuously added to the reaction mixture to maintain the $\mathrm{pH}$ at $10 \sim 11$ during the oxidation. Thus the CNFs obtained by the TEMPO-oxidation have a high crystallinity, mostly uniform widths $(3 \sim 4 \mathrm{~nm})$, and large aspect ratios $(>50)$ compared with other nanocelluloses (Saito et al., 2006; Saito et al., 2007; Shinoda et al., 2012). Moreover, these CNFs can be completely dispersed at the individual nanofibril level in water by electrostatic repulsion and/or osmotic effects due to anionically charged sodium carboxylate groups densely present on the surfaces (Saito \& Isogai, 2004).

Electrospinning is a very simple, reproducible, continuous and scalable one-step technology for the preparation of nanofibers with a controllable morphology from solutions of both natural and synthetic polymers (Bhardwaj \& Kundu, 2010). In this process, an electrical potential is applied between a droplet of a polymer solution, or melt, held at the end of a capillary tube and a grounded target. When the applied electric field overcomes the surface tension of the droplet, a charged jet of polymer solution is ejected and is controlled by the electric field (Chronakis, 2005). Several factors can affect the electrospinning process: a) solution properties such as viscosity, conductivity and surface tension, b) controlled variables like hydrostatic pressure in the capillary, electric po- 
tential at the tip, and the collection screen, and c) ambient parameters including temperature, humidity, and air velocity in the electrospinning chamber (Doshi \& Reneker, 1995). Electrospun polymer nanofibers are increasingly finding a number of applications, such as filtration systems and in medical prosthesis, mainly in grafts and vessels. Other targeted applications include tissue templates, electromagnetic shielding, composite delimitation, and liquid crystal devices. It is worth noting that most of these applications are still within the laboratory settings and have yet to be commercialized (Haung et al., 2003). Electrospun fibers produced from PVA have been widely studied over the last two decades (Zhang et al., 2005; Koski et al., 2004; Son et al., 2005; Rosic et al., 2013). Electrospinning has also been used for preparing cellulose-based nanofibers (Khil et al., 2005; Suwantong et al., 2007; Ahn et al., 2012). In recent years, inclusion of CNWs isolated from native cellulose by sulfuric acid hydrolysis significantly improved the elastic modulus of the electrospun nanofibers of CNW/PVA composites, due to the strong interactions of the hydrogen bonding network (Peresin et al., 2010), and uniform and high quality nanofibers of CNW/PVA blendswere successfully prepared by electrospinning method (Cho et al., 2012).

However, very little work has been done to prepare cellulose-based nanofibers using CNFs isolated by TEMPO oxidation method that provides a good way of separating CNFs by combining with mechanical defibrillation. In this work, we prepared nanofibers of CNF/PVA blends by the electrospinning method, and characterized their morphology, diameter and diameter distribution by scanning electron microscopy.

\section{Experimental}

\subsection{Materials}

A sample of hardwood bleached kraft pulp (HW-BKP) was obtained from Moorim Paper Co. Ltd., Korea and stored in a humidity chamber at $25^{\circ} \mathrm{C}$ before use. TEMPO, sodium bromide $(\mathrm{NaBr})$, sodium hypochlorite $(\mathrm{NaOCl})$ solution and other chemicals were purchased from Sigma-Aldrich and PVA (Mw 66,000) from Duksan Pure Chemical Co., Ltd., Korea, and all chemicals were used as received. PVA solution (10 wt\%) with degrees of hydrolysis between 98 and $99 \%$ was first prepared at $80^{\circ} \mathrm{C}$ with stirring for 6 hours.

\subsection{Isolation of CNFs}

CNFs were isolated from cellulose pulp according to a modified procedure of the TEMPOoxidation method (Saito et al., 2006). In brief, $2 \mathrm{~g}$ of kraft pulp sample was suspended in water $(150 \mathrm{~mL})$ containing TEMPO $(0.025 \mathrm{~g})$ and sodium bromide $(0.25 \mathrm{~g})$. The TEMPO-mediated oxidation of the cellulose slurry was initiated by adding $5 \mathrm{mmol}$ of $13 \% \mathrm{NaClO}$ per gram of cellulose and carried out at room temperature under gentle agitation. The $\mathrm{pH}$ was maintained at 10.5 by adding $0.5 \mathrm{M} \mathrm{NaOH}$. When no more decrease in $\mathrm{pH}$ was observed, the reaction was finished and $\mathrm{pH}$ was adjusted to 7 by adding $0.5 \mathrm{M} \mathrm{HCl}$. The TEMPO-oxidized product was thoroughly washed with water by filtration and physically fibrillated by ultra-sonication with a sonicator (Sonosmasher, Jeio Tech, Korea) for $20 \mathrm{~min}$. The suspension was then centrifuged at about $3,000 \mathrm{~g}$ in two to three times for 30 minutes each. The super- 
natant was decanted and collected as the CNF suspension. The yield of CNFs was calculated as a percentage of the initial weight of $\mathrm{CNF}$ suspension after drying in a drying oven at $105^{\circ} \mathrm{C}$. The final concentration of the CNFs was determined as $0.1 \%$ by weight/volume.

\subsection{Preparation of CNF/PVA Solutions and Viscosity Measurement}

The CNF suspension was first concentrated to $0.2 \%(\mathrm{w} / \mathrm{v})$ by rotary evaporation (Rotovapor R110, BÜCHI, Switzerland) and then mixed with a $10 \mathrm{wt} \%$ PVA solution that had been obtained by vigorous mechanical stirring at $80^{\circ} \mathrm{C}$ for 4 hours, followed by continuous stirring until the solution reached room temperature. CNFs of different contents $(0,1,3$, and $5 \mathrm{wt} \%)$ were added into the PVA solution, and the final mixtures were sonicated for $10 \mathrm{~min}$ (Sonosmasher, Jeio Tech, Korea) at $50 \%$ power output to get homogeneous suspensions. The viscosity of final mixtures at different $\mathrm{CNF}$ concentrations was measured by rheometry (MARS III, Hakke, Germany) with a cone and plate geometry at a shear rate of $10 \mathrm{~s}^{-1}$ and $25^{\circ} \mathrm{C}$. The radius and angle of the cone was $60 \mathrm{~mm}$ and $1^{\circ}$, respectively.

\subsection{Electrospining of CNF/PVA Composite Blends}

The electrospinning of CNF/PVA suspensions was performed with the electrospinning apparatus (NNC-ESP200, NanoNC, Seoul, Korea). The $\mathrm{CNF} / \mathrm{PVA}$ solutions were loaded into a plastic syringe using a 22 gauge stainless steel need leat the tip. The needle was electrified by a high voltage DC supply at an applied voltage of $16 \mathrm{kV}$. The tip to collector distance was 19 cm. After electrospinning, the collected CNF/ PVA composite nanofibers were stored in a desiccator at room temperature.

\subsection{Characterization of CNFs}

\subsubsection{Morphology and Dimension of CNFs}

CNFs were examined and their dimensions measured with a transmission electron microscope (TEM) (H-7600, Hitachi, Japan), operating at an acceleration voltage of $100 \mathrm{kV}$. The sample was diluted to a concentration of $0.01 \%$ w/v. To examine the morphology of the CNFS, three droplets of the suspension were placed on $\mathrm{Cu}$-grids coated with a thin carbon film and then allowed to dry at $30^{\circ} \mathrm{C}$ for $10 \mathrm{~min}$. To enhance the contrast in the TEM, the grids were floated on drops of $3 \%$ solution of uranyl acetate for $3 \mathrm{~min}$ and then dried at $70^{\circ} \mathrm{C}$ for 10 min. The particle dimensions were obtained by measuring 30 individual CNFs on TEM micrographs.

\subsubsection{Morphology and Dimension of Electro- spun CNF/PVA Composite Nanofibers}

The morphology of the CNF/PVA composite nanofibers was investigated using a field emission scanning electron microscope (FE-SEM, S-4300, Hitachi, Japan), operating at an accelerating voltage of $15 \mathrm{kV}$. A small piece of nanofiber mat was placed on carbon applied to stub and coated with $\mathrm{Pt} / \mathrm{Au}$. The average diameter of the composite nanofibers was obtained by measuring 50 individual fibers on FE-SEM micrographs using an image analysis software (Ver. 8.5, IMT i-solution Inc., Canada). 


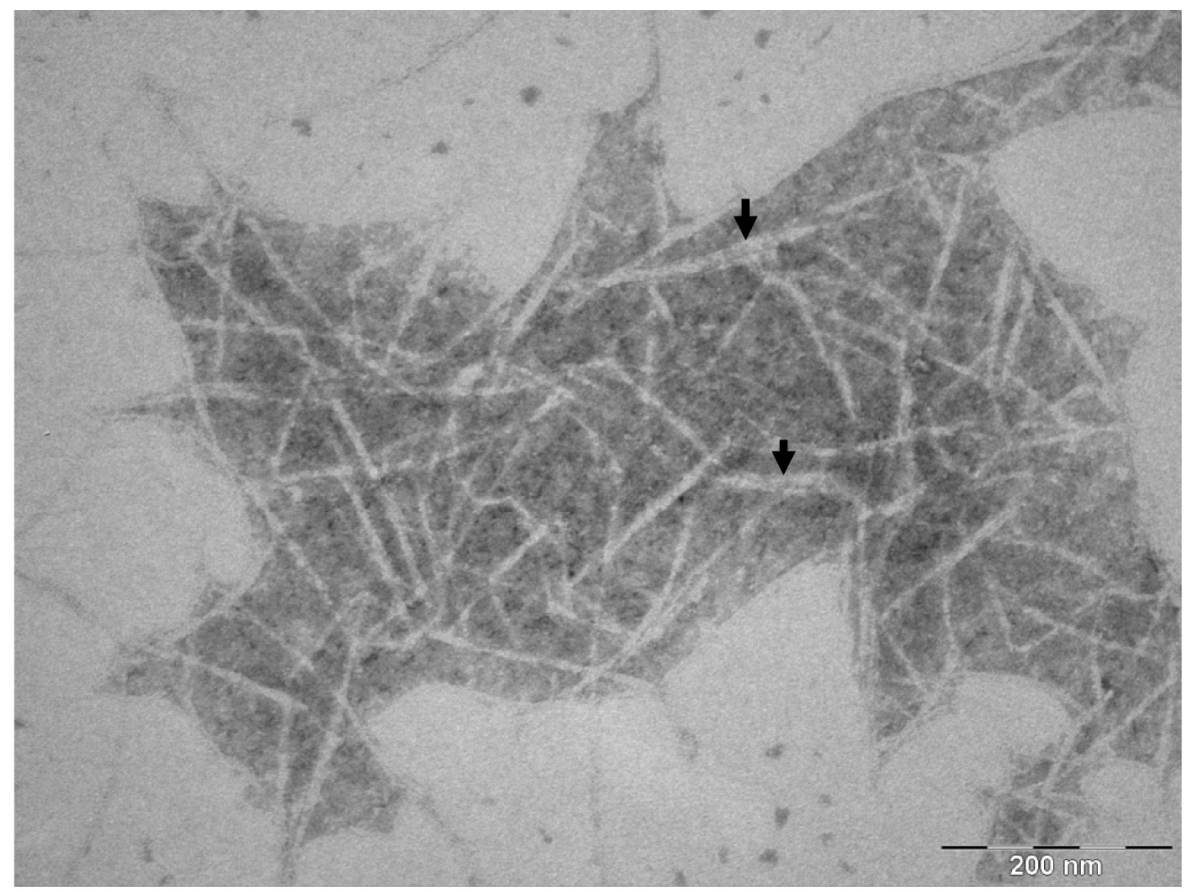

Fig. 1. TEM images of CNFs isolated by TEMPO- Oxidation from HW-BKP. (a) $\times 60,000$, (b) $\times 100,000$.

\section{RESULTS AND DISCUSSION}

\subsection{CNFs' Morphology and Dimension and Viscosity of CNF/PVA Solution}

High magnification TEM images of the CNFs prepared by the TEMPO-oxidation method at different magnification levels are depicted in Fig. 1. While the majority of CNFs were fibrillated, some were not fully fibrillated into nanoscale structure (arrows in Fig. 1). As determined by TEM, the average diameter of fibrillated CNFS was about $7.07 \pm 0.99 \mathrm{~nm}$, a value that matches other measurements (Shinoda et al. 2012). It was considered that the fibrillated CNFs would provide an efficient reinforcement with electrospun nanofibers of CNF/PVA blends, while unfibrillates or macrofibrils cause inconsistencies. Prior to preparing nanofibers by electrospinning, the viscosity of CNF/PVA solutions was determined as a function of CNF content. As expected, the viscosity of the suspension decreased with an increase in the CNF content (Fig. 2) because the amount of water in the $\mathrm{CNF}$ suspension increased with an increase in the $\mathrm{CNF}$ content in the CNF/PVA solution. It is expected that the viscosity of CNF/PVA solution will have an influence on the quality of electrospun nanofibers.

\subsection{Morphology and Dimension of Electro- spun CNF/PVA Nanofibers}

Fig. 3 shows the morphology of the electrospun CNF/PVA composite nanofibers observed by FE-SEM. Figs. 4 and 5 represent average diameter and diameter distribution, respectively, of CNF/PVA composite nanofibers as a func- 


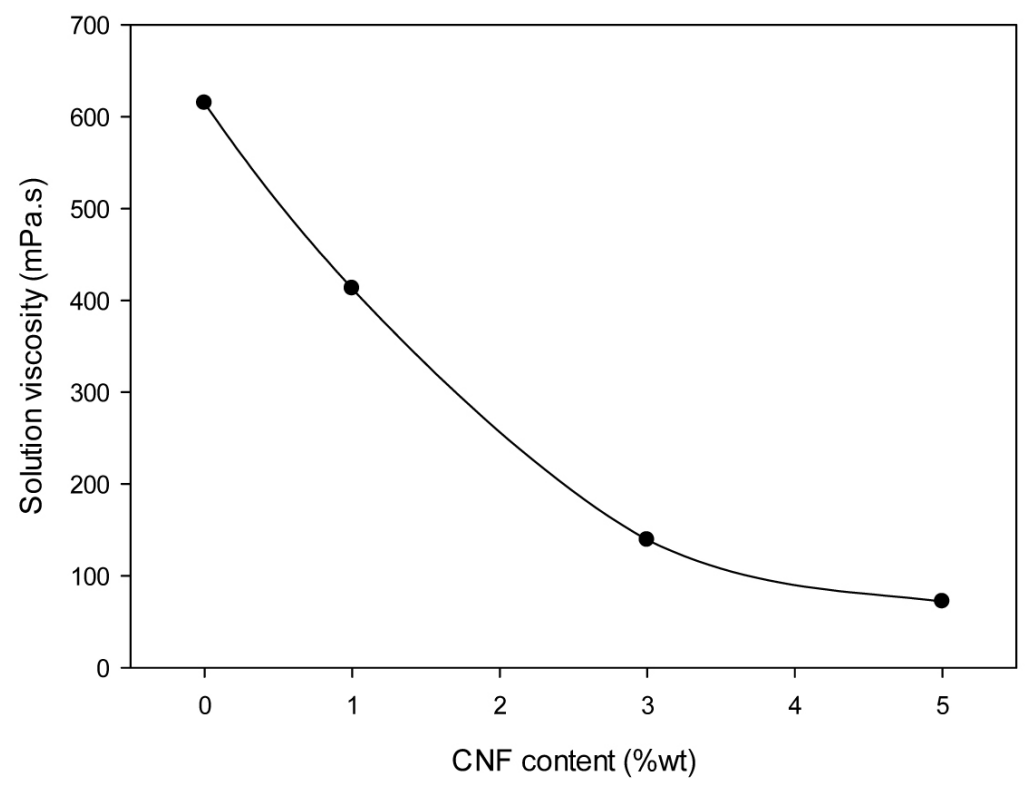

Fig. 2. The viscosity of CNF/PVA solution blends as a function of CNF content.

tion of CNF content. FE-SEM images from (a) to (h) in Fig. 3 display the morphology of the $\mathrm{CNF} / \mathrm{PVA}$ composite nanofibers at different CNF contents $(0 \sim 5 \mathrm{wt} \%)$ after electrospinning. The average diameter of the nanofibers of $\mathrm{CNF} /$ PVA blends decreased as the CNF content increased (Fig. 4). In general, the fiber diameter increased with an increase in the viscosity (Gupta et al., 2005; Mit-uppatham, Mithitanakul, \& Supaphol, 2004), showing a good correlation between the diameter of electrospun fiber and solution viscosity. Other studies have also reported that a higher viscosity resulted in a larger fiber diameter in eletrospinning (Hamad, 2002; Baumgarten, 1991; Doshi \& Reneker, 1995). Therefore, the decrease of fiber diameter with an increase in the CNF content is attributed to the viscosity of dope suspension.

Electrospun nanofibers prepared from the pure PVA solution showed very smooth and uniform nanofibers without any beads (Fig. 3, $a, b)$. The average diameter of the control nanofibers was about $144.7 \mathrm{~nm}$ (Fig. 4). More than $84 \%$ of the nanofibers had diameters, ranging from $120 \mathrm{~nm}$ to $180 \mathrm{~nm}$ (Fig. 5, a).

When $1 \%$ CNFs was added into the PVA solution, the nanofibers of the CNF/PVA blend also showed fairly uniform and high quality nanofibers without any beads (Fig. 3, c and d). The average diameter of the electrospun nanofibers was about $102 \mathrm{~nm}$ (Fig. 4). No beads were observed for the electrospun nanofibers of $1 \% \mathrm{CNF} / \mathrm{PVA}$ blend, and $84 \%$ nanofibers had a diameter range of $80 \sim 120 \mathrm{~nm}$ (Fig. 5, b).

As the CNF content increased to $3 \%$ and $5 \%$, there were elliptical beads formed in the electrospun nanofibers. Measurements of the nanofiber diameter were done for normal nanofibers with no elliptical beads. The number of elliptical beads were relatively small at 3\% $\mathrm{CNF}$ content. However, the majority of the nanofibers were uniform, and the frequency of 

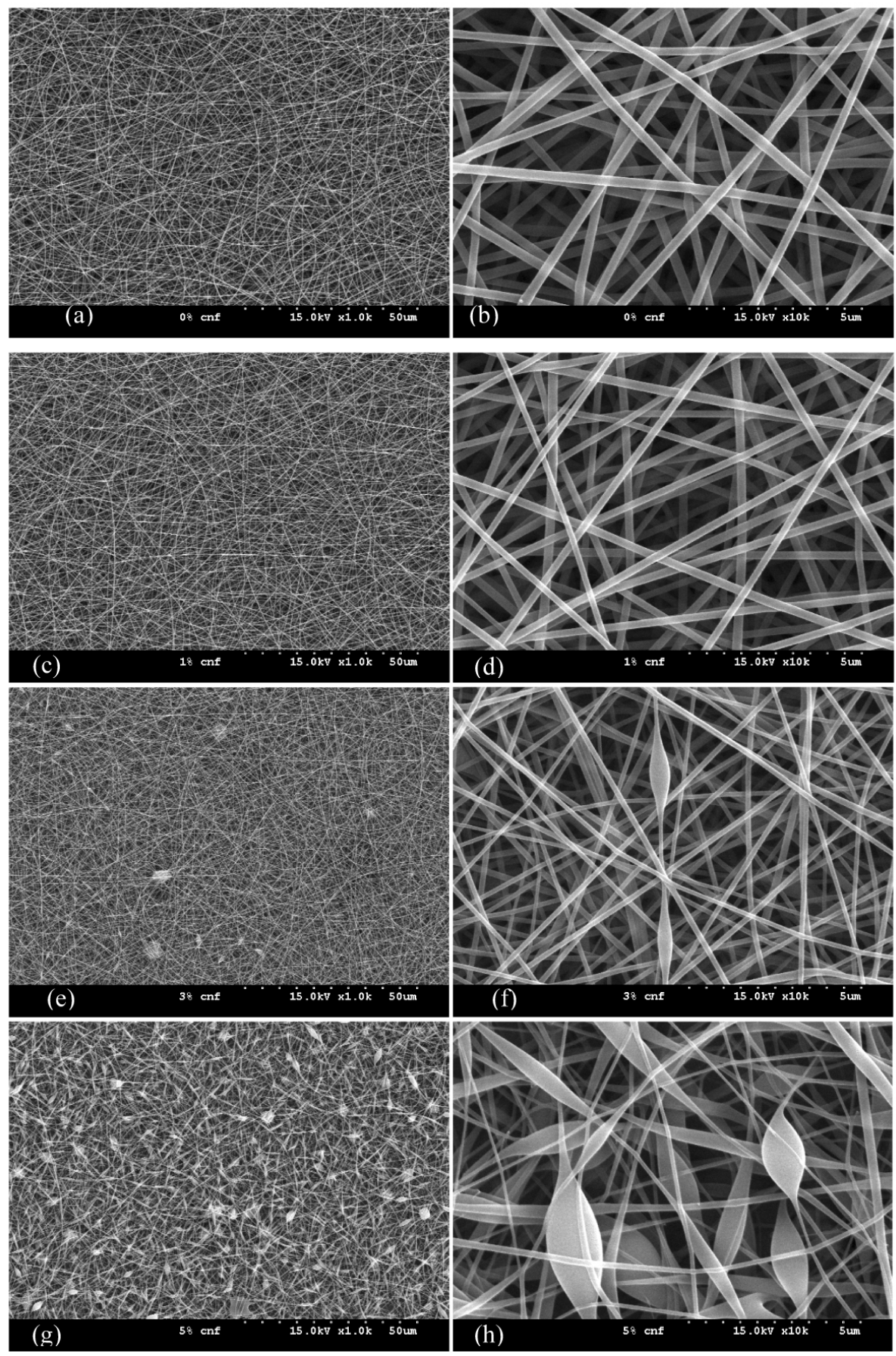

Fig. 3. The FE-SEM images of electrospun nanofibers of CNW/PVA composites at different CNF loadings. (a) $\sim$ (b) $0 \% \mathrm{CNF},($ c) $\sim$ (d) $1 \% \mathrm{CNF}$, (e) (f) $3 \% \mathrm{CNF}$, and (g) (h) $5 \% \mathrm{CNF}$. 


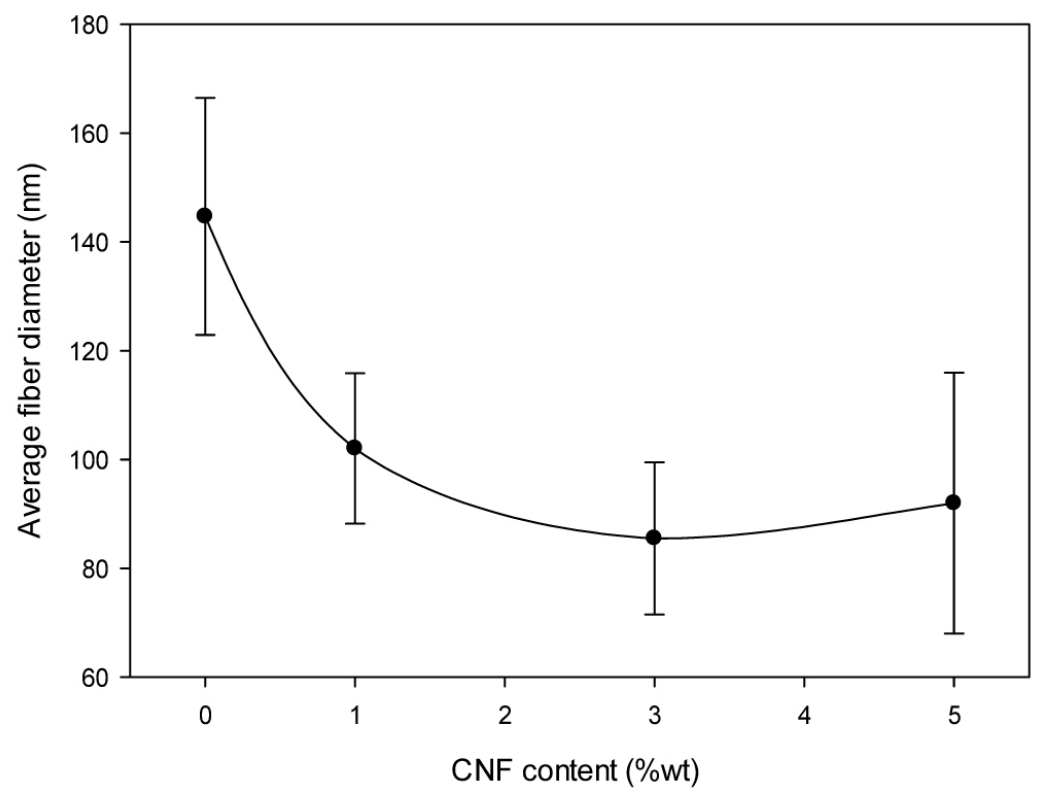

Fig. 4. Average nanofiber diameters with standard deviation of CNFs/PVA composites as a function of $\mathrm{CNF}$ content.

beads was relatively low (Fig. 3, e and f). The average diameter of nanofibers was $85 \mathrm{~nm}$, a valuelower than that for $1 \% \mathrm{CNF}$ content. About $88 \%$ of the electrospun nanofibers were in the range of $60 \sim 80 \mathrm{~nm}$ (Fig. 5, c).

At the highest CNF content of $5 \%$, the electrospun nanofibers contained many elliptical beads with a large variation in the nanofiber diameter (Fig. 3, g and h), and with twisted and irregular forms. There was also a relatively large deviation in the average diameter $(92 \mathrm{~nm})$ of the nanofibers (Fig. 4), which could be attributed to the presence of frequent beads. The diameter of nanofibers widely ranged from 40 $\mathrm{nm}$ to $160 \mathrm{~nm}$ which could explain the large deviation. About $60 \%$ nanofibers of $5 \% \mathrm{CNF} /$ PVA blend were greater than $80 \mathrm{~nm}$ in diameter (Fig. 5, d).

In electrospinning, an electric field is used to create a charged jet of polymer solution. As this jet travels in air, the solvent evaporates leaving behind a charged fiber. So, the electrospinning process is affected by many parameters (Doshi \& Reneker, 1995). The observed beads in the electrospun nanofibers at 3\% and $5 \% \mathrm{CNF}$ content could be related to the applied conductivity and surface tension of the $\mathrm{CNF} /$ PVA suspensions. As the charged jet travels in air, its diameter decreases due to the simultaneous effect of stretching of the jet and evaporation of the solvent. Thus, it was difficult to form a stable jet as the CNF content increased. So, a decrease in the viscosity and PVA content in the CNF/PVA suspension could result in a reduced surface tension of the jet, and consequent production of nanofibers with small diameter as well as elliptical beads. Thus, a decrease in the viscosity of $3 \%$ and $5 \%$ CNF solutions could be mainly responsible for the formation of beaded fibers prepared by electro- 

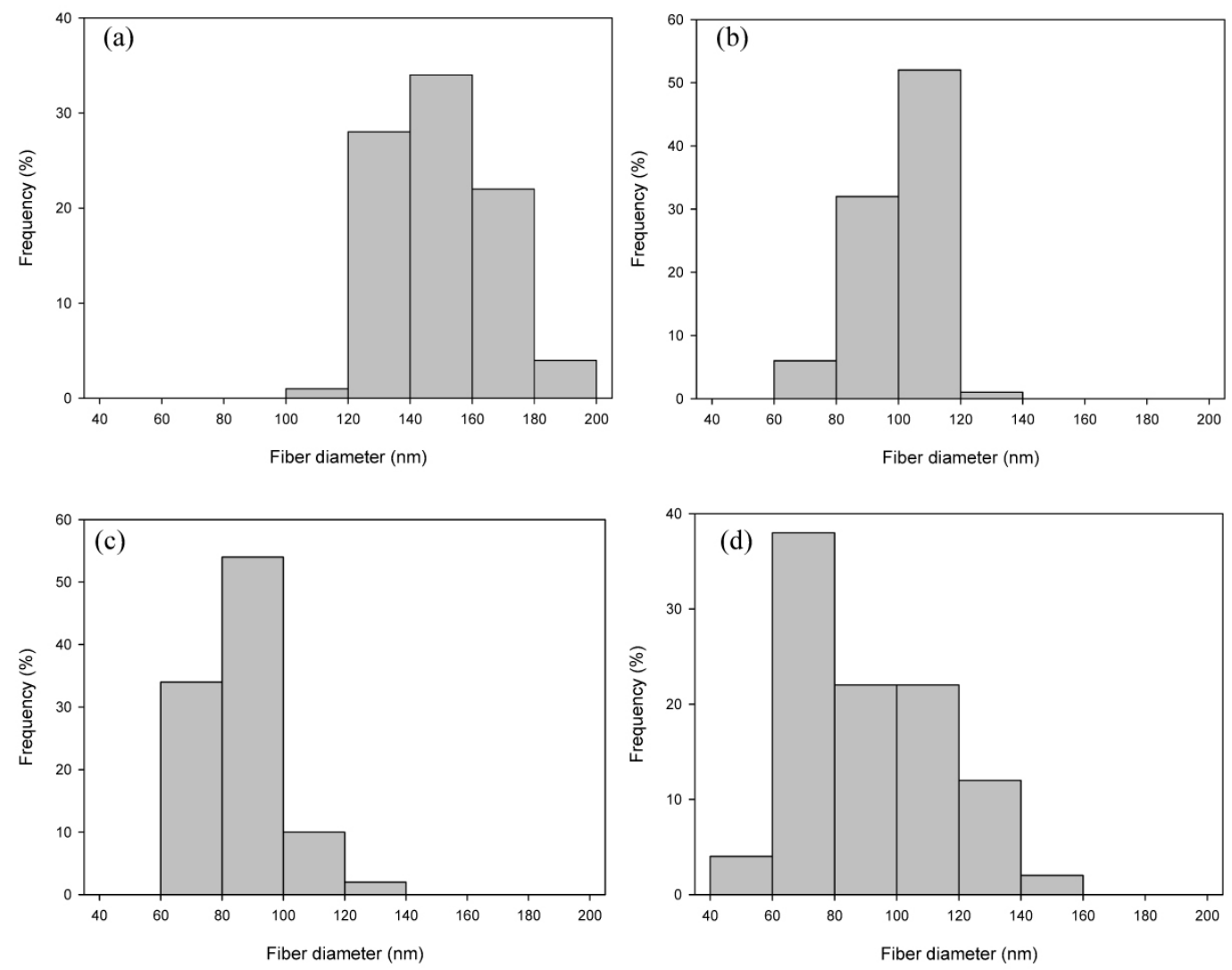

Fig. 5. Diameter distributions of the electrospun $\mathrm{CNF} / \mathrm{PVA}$ composites nanofiber as a function of $\mathrm{CNF}$ content. (a) $0 \% \mathrm{CNF}$, (b) $1 \% \mathrm{CNF}$, (c) $3 \% \mathrm{CNF}$, and (d) $5 \% \mathrm{CNF}$.

spinning, which was compatible with the reported result (Fong, Chun, \& Reneker, 1999). In addition, unfibrillated CNFs (Fig. 1) might also influence the formation of unstable jet which could contribute to the formation of beads at high CNF contents, (3\% and 5\% levels).

\section{CONCLUSION}

This study reports the preparation of cellulose-based polymer nanofibers by electrospinning, using CNFs isolated from native cellulose by TEMPO oxidation and aqueous PVA solution. Morphology and dimension of CNFs were characterized by TEM, while the morphology of the nanofibers was observed by FESEM. In addition, the viscosity of CNF/PVA blend suspension was determined to explain variations in the diameter and its distribution of nanofibers. The following conclusions are drawn from the results obtained:

1) The CNFs as observed with TEM were mainly fibrillated structures in an aggregated form, with an average diameter of about $7.07 \pm$ $0.99 \mathrm{~nm}$. Unfibrillated fiber bundles were also observed in CNFs, which impacted electrospun nanofiber diameter and the formation of beads at high $\mathrm{CNF}$ contents. 
2) Electrospinning was successfully employed to prepare uniform nanofibers from CNF/PVA blends. The viscosity of CNF/PVA suspension and the diameter of nanofibers decreased as the CNF content increased. Nanofiber diameter distribution also followed a similar trend.

3) These results indicate that electrospinning of CNF/PVA solutions can produce uniform nanofibers suitable for a variety of novel and high value applications.

\section{ACKNOWLEDGEMENT}

This work was supported by Kyungpook National University Research Fund, 2011.

\section{REFERENCES}

1. Ahn, Y., Lee, S.H., Kim, H.J., Yang, Y.H., Hong, J.H., Kim, Y.H., and Kim, H. 2012. Electrospinning of lignocellulosic biomass using ionic liquid. Carbohydr. Polym. 88(1): 395 398.

2. Azizi Samir, M.A.S.A., Alloin, F., and Dufresne, A. 2005. Review of recent research into cellulosic whiskers, their properties and their application in nanocomposite field. Biomacromolecules, 6: $612 \sim 626$.

3. Baumgarten, P.K. 1971. Electrostatic spinning of acrylic microfibers. J. Colloid. Interf. Sci. 36: 71 $\sim 79$.

4. de Nooy, A.E.J., Besemer, A.C., and van Bekkum, H. 1995. Highly selective nitroxyl radical-mediated oxidation of primary alcohol groups in water-soluble glucans. Carbohydr. Res. 269: $89 \sim 98$.

5. de Nooy, A.E.J., Besemer, A.C., and van Bekkum, H. 1996.On the use of stable organic nitroxyl radicals for the oxidation of primary and secondary alcohols. Synth. 10: 1153 1174.

6. Bhardwaj, N., and Kundu, S.C. 2010. Electrospinning: a fascinating fiber fabrication technique. Biotechnol. Adv. 28: 325 347.
7. Cho, M., Park, B.D., and Kadla, J.F. 2012. Characterization of Electrospun Nanofibers of Cellulose Nanowhisker/Polyvinyl Alcohol Composites. J. Kor. Wood Sci. Technol. 40(2): $71 \sim 77$.

8. Chronakis, I.S. 2005. Novel nanocomposites and nanoceramics based on polymer nanofibers using electrospinning process-A review. J. Mater. Process. Technol. 167: 283 293.

9. Doshi, J. and Reneker, D.H. 1995. Electrospinning process and applications of electrospun fibers. J. Electrostat. 35: $151 \sim 160$.

10. Eichhorn, S.J., Dufresne. A., Aranguren, M., Marcovich, N.E., Capadona, J.R., Rowan, S.J., Weder, C., Thielemans, W., Roman, M., Renneckar, S., Gindl, W., Veigel, S., Keckes, J., Yano, H., Abe, K., Nogi, M., Nakagaito, A.N., Mangalam, A., Simonsen, J., Benight, A.S., Bismarck, A., Berglund, L.A., and Peijs, T. 2010. Review: current international research into cellulose nanofibres and nanocomposites. J.Mater. Sci. 45: 1 33.

11. Fong H., Chun I., Reneker D.H. 1999. Beaded nanofibers formed during electrospinning, Polymer 40: $4585 \sim 4592$.

12. Fukuzumi, H., Saito, T., Iwata, T., Kumamoto, Y., and Isogai, A. 2009. Transparent and high gas barrier films of cellulose nanofibers prepared by TEMPO-mediated oxidation. Biomacromolecules, 10(1): $162 \sim 165$.

13. Gupta, P., Elkins, C., Long, T.E., and Wilkes, G.L. 2005. Electrospinning of linear homopolymers of poly(methyl methacrylate): exploring relationships between fiber formation, viscosity, molecular weight and concentration in a good solvent. Polym. 46: 4799 4810.

14. Hamad, W. 2002. Cellulosic Materials: Fibers, Networks and Composite, Kluwer Academic Publisher, Massachusetts.

15. Huang, Z.-M., Zhang, Y.-Z., Kotaki, M., and Ramakrishna, S. 2003. A review of polymer nanofibers by electrospinning and their applications in nanocomposites. Compos. Sci. Technol. 63: $2223 \sim 2253$.

16. Isogai, A., Saito, T., and Fukuzumi, H. 2011. 
TEMPO-oxidized cellulose nanofibers Nanoscale, 3: $71 \sim 85$.

17. Khil, M.S., Kim, H.Y., Kang, Y.S., Bang, H.J., and Lee, D.R. 2005. Preparation of electrospun oxidized cellulose mats and their in vitro degradation behavior. Macromol. Res. 13(1): $62 \sim 67$.

18. Koski, A., Yim, K., and Shivkumat, S. 2004. Effect of molecular weight on fibrous PVA produced by electrospinning. Mater. Lett. 58: 493 497.

19. Mit-uppatham, C., Mithitanakul, M., and Supaphol, P. 2004. Ultrafine electrospun polyamide-6 fibers: effect of solution conditions on morphology and average fiber diameter. Macromol. Chem. Phys. 205: 2327 2338.

20. Peresin, M.S., Habibi, Y., Zoppe, J.O., Pawlak, J.J., and Rojas, O.J. 2010. Nanofiber composites of polyvinyl alcohol and cellulose nanocrystals: manufacture and characterization. Biomacromolecules, 11: $674 \sim 681$.

21. Rosic, R., Pelipenko, J., Kristi, J., Kocbek, P., Bester-Rogac, M., and Baumgartner, S. 2013. Physical characteristics of poly(vinyl alcohol) solutions in relation to electrosdpun nanofiber formation. Eur. Polym. J. 49: 290 29.

22. Saito, T., andIsogai, A. 2004. TEMPO-mediated oxidation of native cellulose. The effect of oxidation conditions on chemical and crystal structures of the water-insoluble fractions. Biomacromolecules, 5: $1983 \sim 1989$.

23. Saito, T., Nishiyama, Y., Putaux, J.L., Vignon. M., and Isogai, A. 2006. Homogeneous suspen- sions of individualized microfibrils from TEMPOcatalyzed oxidation of native cellulose. Biomacromolecules, 7(6): 1687 1691.

24. Saito, T., Kimura, S., Nishiyama, Y., and Isogai, A. 2007. Cellulose nanofibers prepared by TEMPO-mediated oxidation of native cellulose. Biomacromolecules, 8: 2485 2491.

25. Shinoda, R., Saito, T., Okita, Y., and Isogai, A. 2012. Relationship between length and degree of polymerization of TEMPO-oxidized cellulose nanofibrils. Biomacromolecules, 13: $842 \sim 849$.

26. Son, W.K., Youk, J.H., and Lee, T.S. 2005. Effect of $\mathrm{pH}$ on electrospinning of poly(vinyl alcohol). Mater. Lett. 59: 1571 $\sim 1575$.

27. Sturcová, A., Davies, G.R., and Eichhorn, S.J. 2005. Elastic modulus and stress transfer properties of tunicate cellulose whiskers. Biomacromolecules, 6: 1055 1061.

28. Suwantong, O., Opanasopit, P., Ruktanonchai, U., and Supaphol, P. 2007. Electrospun cellulose acetate fiber mats containing curcumin and release characteristic of the herbal substance. Polymer 48: 7546 7557.

29. Tashiro, K., and Kobayashi, M. 1991. Theoretical evaluation of three dimensional elastic constants of native and regenerated cellulose: role of hydrogen bonds. Polymer 32: 1516 1526 .

30. Zhang, C., Yuan, X., Wu, L., Han, Y., and Sheng, J. 2005. Study on morphology ofelectrospun poly(vinyl alcohol) mats. Eur. Polym. J. 41(3): $423 \sim 432$. 